Supporting Information for

\title{
Intrinsically Visible Light-Responsive Liquid Crystalline Physical Gels Driven by Halogen Bond
}

Xun Tong ${ }^{\dagger}$, Xiaoyu Zhao ${ }^{\dagger}$, Yuan Qiu*, , Hong Wang ${ }^{\dagger}$, Yonggui Liao ${ }^{* \dagger, \dagger}$, Xiaolin

$$
\mathrm{Xie}^{\dagger, \dagger}
$$

$\uparrow$ Key Laboratory of Material Chemistry for Energy Conversion and Conversion and Storage, Ministry of Education, Hubei Key Laboratory of Material Chemistry and Service Failure, School of Chemistry and Chemical Engineering, Huazhong University of Science and Technology, Wuhan 430074, China

$\$$ State Key Laboratory of Material Processing and Die\&Mould Technology, Huazhong University of Science and Technology, Wuhan 430074, China

E-mails: ygliao@mail.hust.edu.cn (Y. Liao); wukong.s@163.com (Y. Qiu)

Number of pages: 8

Number of tables: 6

Table of Contents

1. Table 1. Calculated infrared spectrum of Azopy- $\mathrm{C}_{10} \quad$ S2

2. Table 2. Calculated infrared spectrum of BTFIPD S3

3. Table 3. Calculated infrared spectrum of 5CB

4. Table 4. Cartesian coordinates of the optimized Azopy- $\mathrm{C}_{10} \quad \mathrm{~S} 4$

5. Table 5. Cartesian coordinates of the optimized BTFIPD S6

$\begin{array}{ll}\text { 6. Table 6. Cartesian coordinates of the optimized 5CB } & \text { S7 }\end{array}$

$\begin{array}{ll}\text { 7. Reference } & \text { S8 }\end{array}$ 
To calculate the infrared spectra of Azopy- $\mathrm{C}_{10}$, BTFIPD and 5CB, density functional theory (DFT) calculations were performed on the Gaussian 09 software package at the M06-2X/6-311G(d,p) level. ${ }^{1}$ The chemical structures were simplified by replacing the alkyl group with methyl group to save the computation cost. Chemical structures were optimized to arrive the stationary point. Analytic frequency calculations were used to judge the stationary point and generate the infrared spectra.

Table S1. Calculated infrared spectrum of Azopy- $\mathrm{C}_{10}$

\begin{tabular}{|c|c|c|c|c|c|}
\hline Mode & $\begin{array}{c}\text { Wavenumber } \\
\left(\mathrm{cm}^{-1}\right)\end{array}$ & Mode & $\begin{array}{c}\text { Wavenumber } \\
\left(\mathrm{cm}^{-1}\right)\end{array}$ & Mode & $\begin{array}{c}\text { Wavenumber } \\
\left(\mathrm{cm}^{-1}\right)\end{array}$ \\
\hline 1 & 410.92 & 32 & 995.20 & 63 & 1467.36 \\
\hline 2 & 427.98 & 33 & 1009.17 & 64 & 1468.91 \\
\hline 3 & 436.65 & 34 & 1009.76 & 65 & 1489.36 \\
\hline 4 & 452.62 & 35 & 1018.46 & 66 & 1507.54 \\
\hline 5 & 496.16 & 36 & 1022.13 & 67 & 1518.37 \\
\hline 6 & 520.75 & 37 & 1028.88 & 68 & 1532.75 \\
\hline 7 & 526.58 & 38 & 1030.94 & 69 & 1548.23 \\
\hline 8 & 538.81 & 39 & 1034.03 & 70 & 1567.40 \\
\hline 9 & 547.65 & 40 & 1084.64 & 71 & 1627.54 \\
\hline 10 & 581.96 & 41 & 1101.13 & 72 & 1650.29 \\
\hline 11 & 626.93 & 42 & 1105.03 & 73 & 1655.54 \\
\hline 12 & 644.38 & 43 & 1113.29 & 74 & 1667.55 \\
\hline 13 & 654.00 & 44 & 1128.28 & 75 & 1677.88 \\
\hline 14 & 674.48 & 45 & 1141.10 & 76 & 1685.91 \\
\hline 15 & 681.38 & 46 & 1172.78 & 77 & 1696.91 \\
\hline 16 & 705.48 & 47 & 1185.53 & 78 & 1866.35 \\
\hline 17 & 742.51 & 48 & 1197.63 & 79 & 3045.64 \\
\hline 18 & 766.71 & 49 & 1210.53 & 80 & 3112.53 \\
\hline 19 & 786.59 & 50 & 1218.16 & 81 & 3182.98 \\
\hline 20 & 809.14 & 51 & 1239.26 & 82 & 3189.61 \\
\hline
\end{tabular}




\begin{tabular}{ll||ll||ll}
21 & 828.16 & 52 & 1262.02 & 83 & 3192.55 \\
22 & 843.44 & 53 & 1270.04 & 84 & 3223.95 \\
23 & 853.09 & 54 & 1288.88 & 85 & 3226.67 \\
24 & 863.53 & 55 & 1303.95 & 86 & 3228.90 \\
25 & 873.79 & 56 & 1317.15 & 87 & 3234.26 \\
26 & 876.60 & 57 & 1318.52 & 88 & 3236.04 \\
27 & 877.76 & 58 & 1329.52 & 89 & 3237.25 \\
28 & 908.86 & 59 & 1346.01 & 90 & 3246.00 \\
29 & 911.22 & 60 & 1354.02 & 91 & 3249.65 \\
30 & 957.91 & 61 & 1363.84 & 92 & 3251.35 \\
31 & 986.71 & 62 & 1456.45 & 93 & 3262.30 \\
\hline
\end{tabular}

Table S2. Calculated infrared spectrum of BTFIPD

\begin{tabular}{|c|c|c|c|c|c|}
\hline Mode & $\begin{array}{c}\text { Wavenumber } \\
\qquad\left(\mathrm{cm}^{-1}\right)\end{array}$ & Mode & $\begin{array}{c}\text { Wavenumber } \\
\left(\mathrm{cm}^{-1}\right)\end{array}$ & Mode & $\begin{array}{c}\text { Wavenumber } \\
\left(\mathrm{cm}^{-1}\right)\end{array}$ \\
\hline 1 & 415.75 & 15 & 701.28 & 29 & 1300.10 \\
\hline 2 & 417.07 & 16 & 723.14 & 30 & 1324.30 \\
\hline 3 & 421.39 & 17 & 761.21 & 31 & 1343.74 \\
\hline 4 & 422.25 & 18 & 781.20 & 32 & 1462.83 \\
\hline 5 & 443.19 & 19 & 795.06 & 33 & 1465.60 \\
\hline 6 & 449.48 & 20 & 828.27 & 34 & 1500.33 \\
\hline 7 & 456.54 & 21 & 876.10 & 35 & 1508.63 \\
\hline 8 & 498.03 & 22 & 1018.00 & 36 & 1563.10 \\
\hline 9 & 512.16 & 23 & 1032.45 & 37 & 1565.11 \\
\hline 10 & 606.49 & 24 & 1105.74 & 38 & 1638.86 \\
\hline 11 & 630.10 & 25 & 1107.67 & 39 & 1671.63 \\
\hline 12 & 645.09 & 26 & 1209.16 & 40 & 1681.92 \\
\hline 13 & 686.20 & 27 & 1211.76 & 41 & 1697.82 \\
\hline 14 & 686.53 & 28 & 1295.76 & 42 & 1702.78 \\
\hline
\end{tabular}


Table S3. Calculated infrared spectrum of 5CB

\begin{tabular}{|c|c|c|c|c|c|}
\hline Mode & $\begin{array}{c}\text { Wavenumber } \\
\left(\mathrm{cm}^{-1}\right)\end{array}$ & Mode & $\begin{array}{c}\text { Wavenumber } \\
\left(\mathrm{cm}^{-1}\right)\end{array}$ & Mode & $\begin{array}{c}\text { Wavenumber } \\
\left(\mathrm{cm}^{-1}\right)\end{array}$ \\
\hline 1 & 409.41 & 22 & 999.20 & 43 & 1496.26 \\
\hline 2 & 412.06 & 23 & 1007.50 & 44 & 1499.49 \\
\hline 3 & 421.10 & 24 & 1013.45 & 45 & 1544.46 \\
\hline 4 & 491.46 & 25 & 1023.88 & 46 & 1576.93 \\
\hline 5 & 496.53 & 26 & 1042.24 & 47 & 1622.86 \\
\hline 6 & 540.63 & 27 & 1065.43 & 48 & 1649.27 \\
\hline 7 & 577.33 & 28 & 1066.49 & 49 & 1686.21 \\
\hline 8 & 590.46 & 29 & 1140.56 & 50 & 1692.07 \\
\hline 9 & 643.35 & 30 & 1146.87 & 51 & 2409.36 \\
\hline 10 & 651.83 & 31 & 1204.91 & 52 & 3063.05 \\
\hline 11 & 663.35 & 32 & 1212.96 & 53 & 3127.95 \\
\hline 12 & 736.43 & 33 & 1239.16 & 54 & 3152.24 \\
\hline 13 & 760.13 & 34 & 1251.70 & 55 & 3193.00 \\
\hline 14 & 792.31 & 35 & 1291.53 & 56 & 3193.67 \\
\hline 15 & 835.70 & 36 & 1314.28 & 57 & 3209.23 \\
\hline 16 & 840.44 & 37 & 1326.04 & 58 & 3210.98 \\
\hline 17 & 867.06 & 38 & 1335.15 & 59 & 3214.39 \\
\hline 18 & 870.54 & 39 & 1339.45 & 60 & 3214.48 \\
\hline 19 & 882.82 & 40 & 1417.79 & 61 & 3230.39 \\
\hline 20 & 983.15 & 41 & 1438.87 & 62 & 3231.66 \\
\hline 21 & 997.06 & 42 & 1459.68 & & \\
\hline
\end{tabular}

Table S4. Cartesian coordinates of the optimized Azopy- $\mathrm{C}_{10}$

\begin{tabular}{ccccc}
\hline Center & Atomic & \multicolumn{3}{c}{ Cartesian coordinates/Angstroms } \\
number & number & $\mathbf{X}$ & $\mathbf{Y}$ & $\mathbf{Z}$ \\
\hline 1 & 7 & -11.357374 & 1.818501 & 1.144562
\end{tabular}




\begin{tabular}{|c|c|c|c|c|}
\hline 2 & 6 & -10.470291 & 1.544125 & 2.105244 \\
\hline 3 & 6 & -9.129753 & 1.275462 & 1.873223 \\
\hline 4 & 6 & -8.681432 & 1.293275 & 0.553688 \\
\hline 5 & 6 & -9.588904 & 1.576908 & -0.458168 \\
\hline 6 & 6 & -10.911204 & 1.831397 & -0.108963 \\
\hline 7 & 7 & -7.337714 & 1.038966 & 0.145751 \\
\hline 8 & 7 & -6.565822 & 0.787369 & 1.083453 \\
\hline 9 & 6 & -5.222909 & 0.531121 & 0.699611 \\
\hline 10 & 6 & -4.343293 & 0.244251 & 1.739932 \\
\hline 11 & 6 & -3.005553 & -0.023610 & 1.486467 \\
\hline 12 & 6 & -2.563205 & 0.005132 & 0.169589 \\
\hline 13 & 6 & -3.433713 & 0.289098 & -0.881523 \\
\hline 14 & 6 & -4.764881 & 0.551932 & -0.620566 \\
\hline 15 & 8 & -1.270530 & -0.316008 & -0.196486 \\
\hline 16 & 6 & -0.202306 & 0.160659 & 0.510491 \\
\hline 17 & 6 & 1.076099 & -0.329457 & -0.051588 \\
\hline 18 & 8 & -0.306685 & 0.885583 & 1.456856 \\
\hline 19 & 6 & 2.261415 & 0.103720 & 0.554377 \\
\hline 20 & 6 & 3.482466 & -0.320650 & 0.078213 \\
\hline 21 & 6 & 3.542207 & -1.192017 & -1.017609 \\
\hline 22 & 6 & 2.364613 & -1.629551 & -1.627782 \\
\hline 23 & 6 & 1.138604 & -1.193509 & -1.139788 \\
\hline 24 & 8 & 4.781728 & -1.552548 & -1.410105 \\
\hline 25 & 6 & 4.901136 & -2.433474 & -2.510511 \\
\hline 26 & 1 & -10.857165 & 1.539784 & 3.119348 \\
\hline 27 & 1 & -8.444923 & 1.058105 & 2.680762 \\
\hline 28 & 1 & -9.256209 & 1.595121 & -1.488038 \\
\hline 29 & 1 & -11.645266 & 2.056480 & -0.876165 \\
\hline 30 & 1 & -4.730069 & 0.234728 & 2.751403 \\
\hline
\end{tabular}




\begin{tabular}{lllll}
31 & 1 & -2.317962 & -0.236380 & 2.291276 \\
32 & 1 & -3.042729 & 0.296611 & -1.891068 \\
33 & 1 & -5.460759 & 0.776055 & -1.417498 \\
34 & 1 & 2.197059 & 0.776085 & 1.401029 \\
35 & 1 & 4.413478 & -0.000493 & 0.527956 \\
36 & 1 & 2.389920 & -2.302074 & -2.473635 \\
37 & 1 & 0.223298 & -1.529971 & -1.608534 \\
38 & 1 & 5.967190 & -2.590039 & -2.653411 \\
39 & 1 & 4.416172 & -3.391765 & -2.302234 \\
40 & 1 & 4.472261 & -1.993507 & -3.415722 \\
\hline
\end{tabular}

Table S5. Cartesian coordinates of the optimized BTFIPD

\begin{tabular}{|c|c|c|c|c|}
\hline \multirow{2}{*}{$\begin{array}{l}\text { Center } \\
\text { number }\end{array}$} & \multirow{2}{*}{$\begin{array}{l}\text { Atomic } \\
\text { number }\end{array}$} & \multicolumn{3}{|c|}{ Cartesian coordinates/Angstroms } \\
\hline & & $\mathbf{X}$ & $\mathbf{Y}$ & $\mathbf{Z}$ \\
\hline 1 & 6 & 4.541324 & -0.035239 & -0.000089 \\
\hline 2 & 6 & 3.928710 & -1.151440 & 0.551788 \\
\hline 3 & 6 & 2.549845 & -1.268598 & 0.548293 \\
\hline 4 & 6 & 1.739307 & -0.261821 & 0.020437 \\
\hline 5 & 6 & 2.362391 & 0.857343 & -0.541436 \\
\hline 6 & 6 & 3.744461 & 0.958165 & -0.553126 \\
\hline 7 & 7 & 0.349943 & -0.514232 & 0.044357 \\
\hline 8 & 7 & -0.349959 & 0.514244 & 0.044349 \\
\hline 9 & 6 & -1.739315 & 0.261832 & 0.019881 \\
\hline 10 & 6 & -2.550053 & 1.268525 & 0.547591 \\
\hline 11 & 6 & -3.928919 & 1.151359 & 0.550567 \\
\hline 12 & 6 & -4.541322 & 0.035229 & -0.001690 \\
\hline 13 & 6 & -3.744250 & -0.958092 & -0.554576 \\
\hline 14 & 6 & -2.362187 & -0.857258 & -0.542375 \\
\hline 15 & 9 & 4.292882 & 2.029975 & -1.111056 \\
\hline 16 & 9 & 1.666641 & 1.821743 & -1.121363 \\
\hline
\end{tabular}




\begin{tabular}{lllll}
17 & 9 & 1.999724 & -2.347991 & 1.081663 \\
18 & 9 & 4.650132 & -2.125555 & 1.090379 \\
19 & 9 & -1.666220 & -1.821575 & -1.122180 \\
20 & 9 & -4.292462 & -2.029830 & -1.112849 \\
21 & 53 & -6.616939 & -0.142398 & -0.004681 \\
22 & 9 & -4.650544 & 2.125394 & 1.089030 \\
23 & 9 & -2.000132 & 2.347848 & 1.081311 \\
24 & 53 & 6.616944 & 0.142371 & -0.002276 \\
\hline
\end{tabular}

Table S6. Cartesian coordinates of the optimized 5CB

\begin{tabular}{|c|c|c|c|c|}
\hline \multirow{2}{*}{$\begin{array}{l}\text { Center } \\
\text { number }\end{array}$} & \multirow{2}{*}{$\begin{array}{l}\text { Atomic } \\
\text { number }\end{array}$} & \multicolumn{3}{|c|}{ Cartesian coordinates/Angstroms } \\
\hline & & $\mathbf{X}$ & $\mathbf{Y}$ & $\mathbf{Z}$ \\
\hline 1 & 6 & -4.766440 & 1.742978 & 0.000718 \\
\hline 2 & 6 & -3.367404 & 1.751657 & -0.010530 \\
\hline 3 & 6 & -2.662239 & 2.943970 & -0.015134 \\
\hline 4 & 6 & -3.353213 & 4.157540 & -0.006555 \\
\hline 5 & 6 & -4.749671 & 4.165813 & 0.005712 \\
\hline 6 & 6 & -5.443912 & 2.967124 & 0.008350 \\
\hline 7 & 6 & -5.515222 & 0.464696 & 0.004313 \\
\hline 8 & 6 & -5.085810 & -0.619580 & -0.764860 \\
\hline 9 & 6 & -5.788990 & -1.816050 & -0.758419 \\
\hline 10 & 6 & -6.939638 & -1.971623 & 0.014741 \\
\hline 11 & 6 & -7.363420 & -0.888936 & 0.785611 \\
\hline 12 & 6 & -6.666697 & 0.311316 & 0.780422 \\
\hline 13 & 1 & -2.828658 & 0.811789 & 0.009657 \\
\hline 14 & 1 & -1.579530 & 2.945275 & -0.015563 \\
\hline 15 & 1 & -5.278711 & 5.110466 & 0.003390 \\
\hline 16 & 1 & -6.527270 & 2.976411 & -0.008747 \\
\hline 17 & 1 & -4.207165 & -0.516322 & -1.391840 \\
\hline 18 & 1 & -5.441965 & -2.643119 & -1.368765 \\
\hline
\end{tabular}




\begin{tabular}{lllll}
19 & 1 & -8.247021 & -0.991141 & 1.406711 \\
20 & 1 & -7.001025 & 1.129190 & 1.408982 \\
21 & 6 & -7.717237 & -3.261265 & -0.003797 \\
22 & 1 & -8.477042 & -3.240728 & -0.790013 \\
23 & 1 & -7.063572 & -4.113139 & -0.196222 \\
24 & 1 & -8.227308 & -3.427318 & 0.946075 \\
25 & 6 & -2.627890 & 5.396764 & -0.010285 \\
26 & 7 & -2.046902 & 6.389407 & -0.013276 \\
\hline
\end{tabular}

\section{Reference}

(1) Frisch, M. J.; Trucks, G. W.; Schlegel, H. B.; Scuseria, G. E.; Robb, M. A.; Cheeseman, J. R.; Scalmani, G.; Barone, V.; Mennucci, B.; Petersson, G. A.; Nakatsuji, H.; Caricato, M.; Li, X.; Hratchian, H. P.; Izmaylov, A. F.; Bloino, J.; Zheng, G.; Sonnenberg, J. L.; Hada, M.; Ehara, M.; Toyota, K.; Fukuda, R.; Hasegawa, J.; Ishida, M.; Nakajima, T.; Honda, Y.; Kitao, O.; Nakai, H.; Vreven, T.; Montgomery, J. A.; Jr. Peralta, J. E.; Ogliaro, F.; Bearpark, M.; Heyd, J. J.; Brothers, E.; Kudin, K. N.; Staroverov, V. N.; Keith, T.; Kobayashi, R.; Normand, J.; Raghavachari, K.; Rendell, A.; Burant, J. C.; Iyengar, S. S.; Tomasi, J.; Cossi, M.; Rega, N.; Millam, J. M.; Klene, M.; Knox, J. E.; Cross, J. B.; Bakken, V.; Adamo, C.; Jaramillo, J.; Gomperts, R.; Stratmann, R. E.; Yazyev, O.; Austin, A. J.; Cammi, R.; Pomelli, C.; Ochterski, J. W.; Martin, R. L.; Morokuma, K.; Zakrzewski, V. G.; Voth, G. A.; Salvador, P.; Dannenberg, J. J.; Dapprich, S.; Daniels, A. D.; Farkas, O.; Foresman, J. B.; Ortiz, J. V.; Cioslowski, J.; Fox, D. J. Gaussion 09 (D.01), Gaussion, Inc., Wallingford CT, 2013. 